\title{
Article \\ Synchronization in Finite-Time Analysis of Clifford-Valued Neural Networks with Finite-Time Distributed Delays
}

\author{
Grienggrai Rajchakit ${ }^{1}$, Ramalingam Sriraman ${ }^{2}$, Chee Peng Lim ${ }^{3}$, Panu Sam-ang ${ }^{4, *}$ (i) \\ and Porpattama Hammachukiattikul ${ }^{5}$ (D) \\ 1 Department of Mathematics, Faculty of Science, Maejo University, Chiang Mai 50290, Thailand; \\ kreangkri@mju.ac.th \\ 2 Department of Mathematics, Thiruvalluvar University, Vellore 632115, India; sriraman225@gmail.com \\ 3 Institute for Intelligent Systems Research and Innovation, Deakin University, \\ Waurn Ponds, VIC 3216, Australia; chee.lim@deakin.edu.au \\ 4 School of Mathematics, Institute of Science, Suranaree University of Technology, \\ Nakhon Ratchasima 30000, Thailand \\ 5 Department of Mathematics, Faculty of Science and Technology, Phuket Rajabhat University, \\ Phuket 83000, Thailand; porpattama@pkru.ac.th \\ * Correspondence: panu.samang@g.sut.ac.th
}

\section{check for} updates

Citation: Rajchakit, G.; Sriraman, R.; Lim, C.P.; Sam-ang, P.;

Hammachukiattikul, P.

Synchronization in Finite-Time

Analysis of Clifford-Valued Neural

Networks with Finite-Time

Distributed Delays. Mathematics 2021,

9, 1163. https://dx.doi.org/

$10.3390 /$ math 9111163

Academic Editors: Theodore E. Simos and Charampos Tsitouras

Received: 14 April 2021

Accepted: 17 May 2021

Published: 21 May 2021

Publisher's Note: MDPI stays neutral with regard to jurisdictional claims in published maps and institutional affiliations.

Copyright: (c) 2021 by the authors. Licensee MDPI, Basel, Switzerland. This article is an open access article distributed under the terms and conditions of the Creative Commons Attribution (CC BY) license (https:/ / creativecommons.org/licenses/by/ $4.0 /)$.

\begin{abstract}
In this paper, we explore the finite-time synchronization of Clifford-valued neural networks with finite-time distributed delays. To address the problem associated with non-commutativity pertaining to the multiplication of Clifford numbers, the original $n$-dimensional Clifford-valued drive and response systems are firstly decomposed into the corresponding $2^{m}$-dimensional real-valued counterparts. On the basis of a new Lyapunov-Krasovskii functional, suitable controller and new computational techniques, finite-time synchronization criteria are formulated for the corresponding real-valued drive and response systems. The feasibility of the main results is verified by a numerical example.
\end{abstract}

Keywords: Clifford-valued neural network; finite-time synchronization; distributed delay; LyapunovKrasovskii fractional

\section{Introduction}

Neural Network (NN) models have been used successfully to solve a variety of tasks, which include optimization, associative memory, signal and image recognition, as well as other dynamic issues. Recently, the dynamic study of NN models has drawn a great deal of interest from numerous researchers, and useful methods for the stability theory of NN models have been reported [1-6]. Specifically, quaternion- and complex-valued NN models have been recently shown to be useful in many fields, including night vision analysis, radar images, polarized signal classification, 3D wind forecasts, and others [7-11]. Recently, many important results have been published concerning different dynamics of the complex-valued and quaternion-valued NN models [12-19]. There are also several studies focusing on stability including finite stability [12], stability analysis [10,13,17], finite-time stabilizability and instabilizability [14], $\mu$-stability, and multistability [18]. However, due to the slow signal propagation, it is inevitable that time delays exist in nearly all kinds of NN models. Time delays are the main source of various dynamics, such as chaos, poor functionality, divergence and instability [1-3,17-19]. As such, NN dynamics studies involving constant or time-varying delays are essential.

Clifford algebra provides a solid principle to solve geometry problems. It has been implemented in many areas, such as neural computing [20-24], and computer and robot vision [25-27]. Clifford-valued NN models present a generalization of real-, complex-, and quaternion-valued NN models. To address the challenges associated with high-dimensional data and spatial geometric transformation, Clifford-valued NN models are superior to real-, 
complex- and quaternion-valued NN models [28-31]. Recently, theoretical and applied studies on Clifford-valued NN models have become a new research subject. However, the dynamic properties of Clifford-valued NN models are usually more complex than those of real-, complex- and quaternion-valued ones. Due to the non-commutativity of multiplication with respect to Clifford numbers, studies on Clifford-valued NN dynamics are still limited [28-35]. By using the linear matrix inequality (LMI) approach, the authors in [28] derived the global exponential stability criteria with respect to delayed Clifford recurrent $\mathrm{NN}$ models. Leveraging the decomposition process, the issue of global asymptotic stability in Clifford-valued NN models was explored in [29]. In [31], the authors studied the presence of globally asymptotic almost automorphic synchronization pertaining to the problem of Clifford-valued RNN models by using suitable feedback controllers. Utilizing the Lyapunov-Krasovskii functional as well as Banach fixed point theorem, the global asymptotic almost periodic synchronization problems for Clifford-valued NN models were examined in [33].

In 1990, the fundamental principle of drive-response synchronization with respect to chaotic systems was introduced [36]. Since then, the issue of synchronization has become an important research topic attracting great attention from researchers, as this phenomenon is applicable in many areas including image processing, neural computing, associative memory, secure communication traffic systems, and others. As a result, a number of approaches for investigating synchronization of different nonlinear and NN models have been developed. The existing literature mostly focuses on long-time synchronization, particularly exponential and asymptotic synchronization [37-39]. In real situations including limited life spans of machines, relatively speaking, synchronization in finite-time is more pertinent, as it occurs naturally [40-46]. As such, the issue of finite-time synchronization pertaining to NN models is studied via several approaches including LKF method [40], LMI method [41], novel controller techniques [42], and others. In [40], the authors explored synchronization issues in finite-time complex-valued RNN models with time-varying delays and discontinuous activation functions. A useful control and analytical techniques for investigating finite-time synchronization of complex-valued NNs with multiple time-varying delays were studied in [44]. Recently, the authors in [45] derived finite-time synchronization pertaining to complex-valued $\mathrm{NN}$ models that incorporate finite-time distributed delays. However, with respect to finite-time distributed delays, the problem of finite-time synchronization associated with Clifford-valued NN models is yet to be well studied.

To the best of our knowledge, there are few papers that deal with the problem of finitetime synchronization of Clifford-valued NN models with finite-time distributed delays. Indeed, this interesting topic is still an open challenge. Therefore, we study the sufficient conditions pertaining to finite-time synchronization of Clifford-valued NN models with time delays in this paper. The main contributions of this paper are as follows:

(1) The finite-time synchronization of Clifford-valued NNs with finite-time distributed delays is investigated for the first time.

(2) By considering an appropriate controller, Lyapunov functional and new computational methods, some sufficient conditions that ascertain the finite-time synchronization of Clifford-valued NN models are derived by decomposing the Clifford-valued NN model into real-valued models.

(3) When Clifford-valued NN model is reduced to real-, complex-, and quaternion-valued ones, the results obtained in this paper are valid as special cases.

(4) A numerical example with simulations is given to support the effectiveness and merits of the theoretical results.

This paper is organized as follows. In Section 2, the proposed Clifford-valued NN model is formally defined. In Sections 3 and 4, respectively, we derive the new sufficient conditions for finite-time synchronization of the considered NN models and present the numerical example and the associated main results. A summary of the main results is given in Section 5. 


\section{Mathematical Fundamentals and Problem Formulation}

\subsection{Notations}

The superscripts of $T$ and $*$ indicate matrix transposition and matrix involution transposition, respectively. A matrix $\mathcal{O}>0(<0)$ denotes a positive (negative) definite matrix, while $\mathbb{A}$ is defined as the Clifford algebra having $m$ generators over real number $\mathbb{R}$. $\mathbb{A}^{n}$ and $\mathbb{R}^{n}$ denote the $n$-dimensional real Clifford vector as well as real vector spaces, respectively. While, $\mathbb{R}^{n \times n}$ and $\mathbb{A}^{n \times n}$ denote the set of all $n \times n$ real matrices and the set of all $n \times n$ real Clifford matrices, respectively. We define the norm of $\mathbb{R}^{n}$ as $\|\boldsymbol{r}\|=\sum_{i=1}^{n}\left|\boldsymbol{r}_{i}\right|$, and for $A=\left(a_{i j}\right)_{n \times n} \in \mathbb{R}^{n \times n}$, denote $\|A\|=\max _{1 \leq i \leq n}\left\{\sum_{j=1}^{n}\left|a_{i j}\right|\right\}$. While $\boldsymbol{r}=\sum_{A} \boldsymbol{r}^{A} e_{A} \in \mathbb{A}$, denote $|\boldsymbol{r}|_{\mathbb{A}}=\sum_{A}\left|\boldsymbol{r}^{A}\right|$, and for $A=\left(a_{i j}\right)_{n \times n} \in \mathbb{A}^{n \times n}$, denote $\|A\|_{\mathbb{A}}=\max _{1 \leq i \leq n}\left\{\sum_{j=1}^{n}\left|a_{i j}\right|_{\mathbb{A}}\right\}$. For $\|\varphi\|_{\tau} \leq \sup _{-\tau \leq s \leq 0}\|\varphi(t+s)\|$.

\subsection{Clifford Algebra}

The definition of Clifford real algebra over $\mathbb{R}^{m}$ is

$$
\mathbb{A}=\left\{\sum_{A \subseteq\{1,2, \ldots, m\}} a^{A} e_{A}, a^{A} \in \mathbb{R}\right\}
$$

where $e_{A}=e_{l_{1}} e_{l_{2}} \ldots e_{l_{v}}$ with $A=\left\{l_{1}, l_{2}, \ldots, l_{v}\right\}, 1 \leq l_{1}<l_{2}<\ldots<l_{v} \leq m$. Moreover $e_{\varnothing}=e_{0}=1$ and $e_{l}=e_{\{l\}}, l=1,2, \ldots, m$ represent the Clifford generators, and they satisfy

$$
\begin{cases}e_{i} e_{j}+e_{j} e_{i}=0, & i \neq j, \quad i, j=1,2, \ldots, m \\ e_{i}^{2}=-1, & i=1,2, \ldots, m \\ e_{0}^{2}=1 . & \end{cases}
$$

For simplicity, when an element is the product of multiple Clifford generators, their subscripts are combined, e.g., $e_{4} e_{5} e_{6} e_{7}=e_{4567}$. Let $\Lambda=\{\varnothing, 1,2, \ldots, A, \ldots, 12 \ldots m\}$, and we have

$$
\mathbb{A}=\left\{\sum_{A} a^{A} e_{A}, a^{A} \in \mathbb{R}\right\},
$$

where $\sum_{A}$ denotes $\sum_{A \in \Lambda}$ and $\mathbb{A}$ is isomorphic to $\mathbb{R}^{2^{m}}$. For any Clifford number $\boldsymbol{r}=\sum_{A} \boldsymbol{r}^{A} e_{A}$, the involution of $\boldsymbol{r}$ is defined by

$$
\overline{\boldsymbol{r}}=\sum_{A} \boldsymbol{r}^{A} \bar{e}_{A}
$$

where $\bar{e}_{A}=(-1)^{\frac{\sigma[A](\sigma[A]+1)}{2}} e_{A}$, and

$$
\sigma[A]= \begin{cases}0, & \text { if } A=\varnothing \\ \nu, & \text { if } A=l_{1} l_{2} \ldots l_{v}\end{cases}
$$

From the definition, we can directly deduce that $e_{A} \bar{e}_{A}=\bar{e}_{A} e_{A}=1$. For a Cliffordvalued function $\boldsymbol{r}=\sum_{A} \boldsymbol{r}^{A} e_{A}: \mathbb{R} \rightarrow \mathbb{A}$, where $\boldsymbol{r}^{A}: \mathbb{R} \rightarrow \mathbb{R}, A \in \Lambda$, we use $\frac{d \boldsymbol{r}(t)}{d t}=$ $\sum_{A} \frac{d \boldsymbol{r}^{A}(t)}{d t} e_{A}$ to represent its derivative. Since $e_{B} \bar{e}_{A}=(-1)^{\frac{\sigma[A](\sigma[A]+1)}{2}} e_{B} e_{A}$, we can write $e_{B} \bar{e}_{A}=e_{C}$ or $e_{B} \bar{e}_{A}=-e_{C}$, where $e_{C}$ is a basis of Clifford algebra $\mathbb{A}$. As an example, 
$e_{l_{1} l_{2}} \bar{e}_{l_{2} l_{3}}=-e_{l_{1} l_{2}} e_{l_{2} l_{3}}=-e_{l_{1}} e_{l_{2}} e_{l_{2}} e_{l_{3}}=-e_{l_{1}}(-1) e_{l_{3}}=e_{l_{1}} e_{l_{3}}=e_{l_{1} l_{3}}$. Therefore, a unique basis $e_{C}$ with respect to a given $e_{B} \bar{e}_{A}$ can be identified. Define

$$
\sigma[B . \bar{A}]= \begin{cases}0, & \text { if } e_{B} \bar{e}_{A}=e_{C}, \\ 1, & \text { if } e_{B} \bar{e}_{A}=-e_{C},\end{cases}
$$

and then, $e_{B} \bar{e}_{A}=(-1)^{\sigma[B . \bar{A}]} e_{C}$.

Moreover, for any $\mathscr{G} \in \mathbb{A}$, there is a unique $\mathscr{G}^{C}$ that satisfies $\mathscr{G}^{B \cdot \bar{A}}=(-1)^{\sigma[B \cdot \bar{A}]} \mathscr{G}^{C}$ for $e_{B} \bar{e}_{A}=(-1)^{\sigma[B . \bar{A}]} e_{C}$. Therefore

$$
\mathscr{G}^{B . \bar{A}} e_{B} \bar{e}_{A}=\mathscr{G}^{B \cdot \bar{A}}(-1)^{\sigma[B \cdot \bar{A}]} e_{C}=(-1)^{\sigma[B \cdot \bar{A}]} \mathscr{G}^{C}(-1)^{\sigma[B . \bar{A}]} e_{C}=\mathscr{G}^{C} e_{C} .
$$

and $\mathscr{G}=\sum_{C} \mathscr{G} e_{e_{C}} \in \mathbb{A}$.

\subsection{Problem Definition}

A class of Clifford-valued NN model with discrete-time-varying delays and distributed delays are considered as follows:

$$
\begin{aligned}
\dot{\boldsymbol{r}}_{i}(t)= & -d_{i} \boldsymbol{r}_{i}(t)+\sum_{j=1}^{n} a_{i j} h_{j}\left(\boldsymbol{r}_{j}(t)\right)+\sum_{j=1}^{n} b_{i j} h_{j}\left(\boldsymbol{r}_{j}\left(t-\tau_{j}(t)\right)\right) \\
& +\sum_{j=1}^{n} c_{i j} \int_{t-\pi_{j}}^{t} \theta_{i j}(t-s) h_{j}\left(\boldsymbol{r}_{j}(s)\right) d s+k_{i}, t \geq 0, \\
\boldsymbol{r}_{i}(t)= & \varphi_{i}(t) \in \mathscr{C}\left((-\infty, 0], \mathbb{A}^{n}\right),
\end{aligned}
$$

where $i \in N, j \in N(N=1,2, \ldots, n)$, and $n$ corresponds to the number of neurons; $\boldsymbol{r}_{i}(t) \in \mathbb{A}$ represents the state vector of the $i$ th unit; $d_{i} \in \mathbb{R}^{+}$indicates the rate that the $i$ th unit is going to reset its potential to the resting state in isolation in the event that it is not connected to the network and external inputs; $a_{i j}, b_{i j}, c_{i j} \in \mathbb{A}$ indicate the strengths of connection weights; $k_{i} \in \mathbb{A}$ is the external input associated with the $i$ th unit; $h_{j}(\cdot): \mathbb{A}^{n} \rightarrow \mathbb{A}^{n}$ is the activation function pertaining to signal transmission; $\tau_{j}(t)$ indicates the bounded function and $\pi_{j}$ is a constant; $\theta_{i j}(t)$ is a non-negative bounded scalar function defined on $[0,+\infty)$ describing the delay kernel of the time distributed delay along the axon of the $j$ th unit from the $i$ th unit; $\varphi(t)=\left[\varphi_{1}(t), \ldots, \varphi_{n}(t)\right]^{T}$ is the initial value in $\mathscr{C}\left((-\infty, 0], \mathbb{A}^{n}\right)$, which denotes the Banach space of all continuous functions mapping $(-\infty, 0]$ into $\mathbb{A}^{n}$.

Assumption 1. Positive constants $\tau_{j}$ and $\mu_{j}<1$ exist, whereby $0<\tau_{j}(t) \leq \tau_{j}, \dot{\tau}_{j}(t) \leq \mu_{j}$, $j \in N$.

Remark 1. It is clear that NN model (1) includes real-, complex- and quaternion-valued NN models. These mean that the proposed NN model is more general than the corresponding one in the existing articles. For example, the Clifford-valued NN model (1) includes real-valued $(m=0)$, complex-valued $(m=1)$, and quaternion-valued $(m=2)$ counterparts as its special cases.

We take (1) as the drive system. The corresponding response system is constructed as follows:

$$
\begin{aligned}
\dot{\boldsymbol{s}}_{i}(t)= & -d_{i} \boldsymbol{s}_{i}(t)+\sum_{j=1}^{n} a_{i j} h_{j}\left(\boldsymbol{s}_{j}(t)\right)+\sum_{j=1}^{n} b_{i j} h_{j}\left(\boldsymbol{s}_{j}\left(t-\tau_{j}(t)\right)\right) \\
& +\sum_{j=1}^{n} c_{i j} \int_{t-\pi_{j}}^{t} \theta_{i j}(t-s) h_{j}\left(\boldsymbol{s}_{j}(s)\right) d s+k_{i}+u_{i}(t), t \geq 0, \\
\boldsymbol{s}_{i}(t)= & \phi_{i}(t) \in \mathscr{C}\left((-\infty, 0], \mathbb{A}^{n}\right),
\end{aligned}
$$


where $i \in N, j \in N(N=1,2, \ldots, n)$, and $n$ corresponds to the number of neurons; $\boldsymbol{s}_{i}(t) \in \mathbb{A}$ represents the state vector of the $i$ th unit; while $\phi(t)=\left[\phi_{1}(t), \ldots, \phi_{n}(t)\right]^{T}$ is the initial value, $\mathscr{C}\left((-\infty, 0], \mathbb{A}^{n}\right)$ denotes the Banach space of all continuous functions mapping $(-\infty, 0]$ into $\mathbb{A}^{n}$. In addition, the control input is indicated by $u_{i}(t)$, while other notations associated with (3) and (4) are the same as those in (1) and (2).

Assumption 2. Function $h_{j}(\cdot)$ fulfills the Lipschitz continuity condition with respect to the ndimensional Clifford vector. Given each $j \in N$, there exists positive constant $l_{j}$ such that for any $x, y \in \mathbb{A}$,

$$
\left|h_{j}(x)-h_{j}(y)\right|_{\mathbb{A}} \leq l_{j}|x-y|_{\mathbb{A}}, j \in N,
$$

where $l_{j}(j \in N)$ is known as the Lipschitz constant and $h_{j}(0)=0$. In addition, positive constant $l_{j}$ exists such that $|h(x)|_{\mathbb{A}} \leq l_{j}$ for any $x \in \mathbb{A}$.

Assumption 3. There exists positive constant $\tilde{\theta}_{i j}$ such that

$$
\int_{0}^{+\infty} \theta_{i j}(s) d s=\tilde{\theta}_{i j}, i, j \in N
$$

\section{Main Results}

We transform the Clifford-valued NN models (1) and (2) into the real-valued models, as to handle non-commutativity of multiplication in Clifford numbers. This can be achieved with the help of $e_{A} \bar{e}_{A}=\bar{e}_{A} e_{A}=1$ and $e_{B} \bar{e}_{A} e_{A}=e_{B}$. Given any $\mathscr{G} \in \mathbb{A}$, a unique $\mathscr{G}^{C}$ that is able to satisfy $\mathscr{G} C e_{C} h^{A} e_{A}=(-1)^{\sigma[B . \bar{A}]} \mathscr{G} C h^{A} e_{B}=\mathscr{G} B \cdot \bar{A} h^{A} e_{B}$ can be identified. By decomposing (1) and (2) into $\dot{\boldsymbol{r}}=\sum_{A} \dot{\boldsymbol{r}}^{A} e_{A}$, we have the following real-valued models:

$$
\begin{aligned}
\dot{\boldsymbol{r}}_{i}^{A}(t)= & -d_{i} \boldsymbol{r}_{i}^{A}(t)+\sum_{j=1}^{n} \sum_{B \in \Lambda} a_{i j}^{A . \bar{B}} h_{j}^{B}\left(\boldsymbol{r}_{j}(t)\right)+\sum_{j=1}^{n} \sum_{B \in \Lambda} b_{i j}^{A . \bar{B}} h_{j}^{B}\left(\boldsymbol{r}_{j}\left(t-\tau_{j}(t)\right)\right) \\
& +\sum_{j=1}^{n} \sum_{B \in \Lambda} c_{i j}^{A . \bar{B}} \int_{t-\pi_{j}}^{t} \theta_{i j}(t-s) h_{j}^{B}\left(\boldsymbol{r}_{j}(s)\right) d s+k_{i}^{A}, t \geq 0, \\
\boldsymbol{r}_{i}^{A}(t)= & \varphi_{i}^{A}(t) \in \mathscr{C}\left((-\infty, 0], \mathbb{A}^{n}\right),
\end{aligned}
$$

where

$$
\begin{aligned}
\boldsymbol{r}^{A}(t)= & \left(\boldsymbol{r}_{1}^{A}(t), \boldsymbol{r}_{2}^{A}(t), \ldots, \boldsymbol{r}_{n}^{A}(t)\right)^{T}, \boldsymbol{r}_{i}(t)=\sum_{A \in \Lambda} \boldsymbol{r}_{i}^{A}(t) e_{A}, \\
k^{A}= & \left(k_{1}^{A}, k_{2}^{A}, \ldots, k_{n}^{A}\right)^{T}, k_{i}=\sum_{A \in \Lambda} k_{i}^{A} e_{A}, \\
h^{B}(\boldsymbol{r}(t))= & \left(h_{1}^{B}\left(\boldsymbol{r}_{1}^{C_{1}}(t), \boldsymbol{r}_{1}^{C_{2}}(t), \ldots, \boldsymbol{r}_{1}^{C_{2} m}(t)\right), h_{2}^{B}\left(\boldsymbol{r}_{2}^{C_{1}}(t), \boldsymbol{r}_{2}^{C_{2}}(t), \ldots, \boldsymbol{r}_{2}^{C_{2} m}(t)\right),\right. \\
& \left.\ldots, h_{n}^{B}\left(\boldsymbol{r}_{n}^{C_{1}}(t), \boldsymbol{r}_{n}^{C_{2}}(t), \ldots, \boldsymbol{r}_{n}^{C_{2}}(t)\right)\right)^{T}, \\
h^{B}(\boldsymbol{r}(t-\tau(t)))= & \left(h_{1}^{B}\left(\boldsymbol{r}_{1}^{C_{1}}(t-\tau(t)), \boldsymbol{r}_{1}^{C_{2}}(t-\tau(t)), \ldots, \boldsymbol{r}_{1}^{C_{2} m}(t-\tau(t))\right),\right. \\
& h_{2}^{B}\left(\boldsymbol{r}_{2}^{C_{1}}(t-\tau(t)), \boldsymbol{r}_{2}^{C_{2}}(t-\tau(t)), \ldots, \boldsymbol{r}_{2}^{C_{2} m}(t-\tau(t))\right), \\
& \left.\ldots, h_{n}^{B}\left(\boldsymbol{r}_{n}^{C_{1}}(t-\tau(t)), \boldsymbol{r}_{n}^{C_{2}}(t-\tau(t)), \ldots, \boldsymbol{r}_{n}^{C_{2} m}(t-\tau(t))\right)\right)^{T}, \\
a_{i j}= & \sum_{C \in \Lambda} a_{i j}^{C} e_{C}, a_{i j}^{A . \bar{B}}=(-1)^{\sigma[A . \bar{B}]} a_{i j}^{C}, \\
b_{i j}= & \sum_{C \in \Lambda} b_{i j}^{C} e_{C}, b_{i j}^{A . \bar{B}}=(-1)^{\sigma[A . \bar{B}]} b_{i j}^{C}, \\
c_{i j}= & \sum_{C \in \Lambda} c_{i j}^{C} e_{C}, c_{i j}^{A . \bar{B}}=(-1)^{\sigma[A . \bar{B}]} c_{i j}^{C}, \\
e_{A} \bar{e}_{B}= & (-1)^{\sigma[A . \bar{B}]} e_{C} .
\end{aligned}
$$


We can use the same method to transform (3) and (4) into the following realvalued models:

$$
\begin{aligned}
\dot{\boldsymbol{s}}_{i}^{A}(t)= & -d_{i} \boldsymbol{s}_{i}^{A}(t)+\sum_{j=1}^{n} \sum_{B \in \Lambda} a_{i j}^{A} \cdot \bar{B} h_{j}^{B}\left(\boldsymbol{s}_{j}(t)\right)+\sum_{j=1}^{n} \sum_{B \in \Lambda} b_{i j}^{A} \cdot \bar{B} h_{j}^{B}\left(\boldsymbol{s}_{j}\left(t-\tau_{j}(t)\right)\right) \\
& +\sum_{j=1}^{n} \sum_{B \in \Lambda} c_{i j}^{A . \bar{B}} \int_{t-\pi_{j}}^{t} \theta_{i j}(t-s) h_{j}^{B}\left(\boldsymbol{s}_{j}(s)\right) d s+k_{i}^{A}+u_{i}^{A}(t), t \geq 0, \\
\boldsymbol{s}_{i}^{A}(t)= & \phi_{i}(t) \in \mathscr{C}\left((-\infty, 0], \mathbb{A}^{n}\right),
\end{aligned}
$$

where

$$
\begin{aligned}
\boldsymbol{s}^{A}(t)= & \left(\boldsymbol{s}_{1}^{A}(t), \boldsymbol{s}_{2}^{A}(t), \ldots, \boldsymbol{s}_{n}^{A}(t)\right)^{T}, \boldsymbol{s}_{i}(t)=\sum_{A \in \Lambda} \boldsymbol{s}_{i}^{A}(t) e_{A}, \\
u^{A}(t)= & \left(u_{1}^{A}(t), u_{2}^{A}(t), \ldots, u_{n}^{A}(t)\right)^{T}, u_{i}(t)=\sum_{A \in \Lambda} u_{i}^{A}(t) e_{A}, \\
h^{B}(s(t))= & \left(h_{1}^{B}\left(\boldsymbol{s}_{1}^{C_{1}}(t), \boldsymbol{s}_{1}^{C_{2}}(t), \ldots, \boldsymbol{s}_{1}^{C_{2} m}(t)\right), h_{2}^{B}\left(\boldsymbol{s}_{2}^{C_{1}}(t), \boldsymbol{s}_{2}^{C_{2}}(t), \ldots, \boldsymbol{s}_{2}^{C_{2} m}(t)\right),\right. \\
& \left.\left.\ldots, h_{n}^{B}\left(\boldsymbol{s}_{n}^{C_{1}}(t), \boldsymbol{s}_{n}^{C_{2}}(t), \ldots, \boldsymbol{s}_{n}^{C_{2}}(t)\right)\right)\right)^{T}, \\
h^{B}(\boldsymbol{s}(t-\tau(t)))= & \left(h_{1}^{B}\left(\boldsymbol{s}_{1}^{C_{1}}(t-\tau(t)), \boldsymbol{s}_{1}^{C_{2}}(t-\tau(t)), \ldots, \boldsymbol{s}_{1}^{C_{2} m}(t-\tau(t))\right),\right. \\
& h_{2}^{B}\left(\boldsymbol{s}_{2}^{C_{1}}(t-\tau(t)), \boldsymbol{s}_{2}^{C_{2}}(t-\tau(t)), \ldots, \boldsymbol{s}_{2}^{C_{2}}(t-\tau(t))\right), \\
& \left.\ldots, h_{n}^{B}\left(\boldsymbol{s}_{n}^{C_{1}}(t-\tau(t)), \boldsymbol{s}_{n}^{C_{2}}(t-\tau(t)), \ldots, \boldsymbol{s}_{n}^{C_{2} m}(t-\tau(t))\right)\right)^{T} .
\end{aligned}
$$

Note that the remaining notations of (9) and (10) are the same as those in (7) and (8).

The error vectors between the real-valued drive models (7) and (8) and the real-valued response models (9) and (10) are defined as $\boldsymbol{e}_{i}^{A}(t)=\boldsymbol{s}_{i}^{A}(t)-\boldsymbol{r}_{i}^{A}(t)$ and $\psi_{i}^{A}(t)=\phi_{i}^{A}(t)-$ $\varphi_{i}^{A}(t)$, respectively. As such, from (7)-(10), the following error models are produced:

$$
\begin{aligned}
\dot{\boldsymbol{e}}_{i}^{A}(t)= & -d_{i} \boldsymbol{e}_{i}^{A}(t)+\sum_{j=1}^{n} \sum_{B \in \Lambda} a_{i j}^{A . \bar{B}}\left(h_{j}^{B}\left(\boldsymbol{s}_{j}(t)\right)-h_{j}^{B}\left(\boldsymbol{r}_{j}(t)\right)\right) \\
& +\sum_{j=1}^{n} \sum_{B \in \Lambda} b_{i j}^{A . \bar{B}}\left(h_{j}^{B}\left(\boldsymbol{s}_{j}\left(t-\tau_{j}(t)\right)\right)-h_{j}^{B}\left(\boldsymbol{r}_{j}\left(t-\tau_{j}(t)\right)\right)\right) \\
& +\sum_{j=1}^{n} \sum_{B \in \Lambda} c_{i j}^{A . \bar{B}} \int_{t-\pi_{j}}^{t} \theta_{i j}(t-s)\left(h_{j}^{B}\left(\boldsymbol{s}_{j}(s)\right)-h_{j}^{B}\left(\boldsymbol{r}_{j}(s)\right)\right) d s+u_{i}^{A}(t), t \geq 0, \\
\boldsymbol{e}_{i}^{A}(t)= & \psi_{i}^{A}(t) \in\left((-\infty, 0], \mathbb{A}^{n}\right) .
\end{aligned}
$$

The following definitions and lemmas are utilized for solving main results in this study.

Definition 2. Ref. [45] Given an appropriate controller, if constant $t_{1}>0$, such that $\left\|\boldsymbol{e}^{A}\left(t_{1}\right)\right\|_{1}=0$ and $\left\|\boldsymbol{e}^{A}(t)\right\|_{1} \equiv 0$ for $t>t_{1}$, where $\left\|\boldsymbol{e}^{A}(t)\right\|_{1}=\sum_{i=1}^{n} \sum_{A \in \Lambda}\left|\boldsymbol{e}_{i}^{A}(t)\right|$, then models (7) and (8) is said to be finite-time synchronization with models (9) and (10).

\section{Finite Time Synchronization}

In accordance with Definition (2), the finite-time synchronization arisen from models (7)-(10) is equal to the finite-time stabilization with respect to the error models (11) and (12) at zero, respectively. As a result, controller $u_{i}(t)$ should satisfy the condition in which $u_{i}(t)=0$ when $\boldsymbol{e}_{i}(t)=0, i \in N$. Inspired by the controllers in [47], and [46], where the sign function is utilized to finite-timely synchronize complex networks without delay, we design the following discontinuous controllers: 


$$
\begin{aligned}
u_{i}(t) & =\sum_{A \in \Lambda} u_{i}^{A}(t) e_{A}, \\
u_{i}^{A}(t) & =-\alpha_{i} \boldsymbol{e}_{i}^{A}(t)-\beta_{i} \operatorname{sgn}\left(\left|\boldsymbol{e}_{i}^{A}(t)\right|\right),
\end{aligned}
$$

where $i \in N, A \in \Lambda, \alpha_{i}>0$ are the control gains, while $\beta_{i}>0$ indicate the constants that need to be tuned. Therefore, the following main result can be obtained.

Remark 3. The controller design (13) is very simple and straightforward. This section demonstrates that the control law (13) can finite-timely synchronize NNs (11) with time-varying discrete delay and bounded time distributed delay.

Theorem 4. Given positive constants $\alpha_{i}, \beta_{i}, i \in N$ and Assumptions 1-3 are satisfied, we have

$$
\begin{aligned}
d_{i}+\alpha_{i}- & \left(\sum_{i=1}^{n} \sum_{j=1}^{n} \sum_{A \in \Lambda} \sum_{B \in \Lambda} l_{j}\left|a_{i j}^{A \cdot \bar{B}}\right|+\sum_{i=1}^{n} \sum_{j=1}^{n} \sum_{A \in \Lambda} \sum_{B \in \Lambda} \frac{1}{1-\mu_{j}} l_{j}\left|b_{i j}^{A \cdot \bar{B}}\right|\right. \\
& \left.+\sum_{i=1}^{n} \sum_{j=1}^{n} \sum_{A \in \Lambda} \sum_{B \in \Lambda} l_{j}\left|c_{i j}^{A . \bar{B}}\right| \tilde{\theta}_{i j}\right)>0, i, j \in N
\end{aligned}
$$

then the Clifford-valued NN models (7) and (8) are synchronized with the NN models (9) and (10) in finite-time under controller (13).

Proof. The following Lyapunov-Krasovskii functional is considered:

$$
\mathscr{V}(t)=\sum_{i=1}^{3} \mathscr{V}_{i}(t),
$$

where

$$
\begin{aligned}
& \mathscr{V}_{1}(t)=\sum_{i=1}^{n} \sum_{A \in \Lambda}\left|\boldsymbol{e}_{i}^{A}(t)\right| \\
& \mathscr{V}_{2}(t)=\sum_{i=1}^{n} \sum_{j=1}^{n} \sum_{A \in \Lambda} \sum_{B \in \Lambda} \frac{1}{1-\mu_{j}}\left|b_{i j}^{A \cdot \bar{B}}\right| l_{j} \int_{t-\tau_{j}(t)}^{t}\left(\boldsymbol{e}_{i}^{A}(s)\right) d s, \\
& \mathscr{V}_{3}(t)=\sum_{i=1}^{n} \sum_{i=1}^{n} \sum_{A \in \Lambda} \sum_{B \in \Lambda}\left|c_{i j}^{A \cdot \bar{B}}\right| l_{j} \int_{-\pi_{j}}^{0} \int_{t+s}^{t} \theta_{i j}(-s)\left(\boldsymbol{e}_{i}^{A}(u)\right) d u d s .
\end{aligned}
$$

We then bring controller (13) into error models (11) and (12). Through the computation of the derivative pertaining to $\mathscr{V}(t)$ with the trajectory of (11), we can derive from Assumption 2 that

$$
\dot{\mathscr{V}}(t)=\sum_{i=1}^{3} \dot{\mathscr{V}}_{i}(t)
$$

where 


$$
\begin{aligned}
& \dot{\mathscr{V}}_{1}(t)=\sum_{i=1}^{n} \sum_{A \in \Lambda} \operatorname{sgn}\left(\boldsymbol{e}_{i}^{A}(t)\right) \dot{\boldsymbol{e}}_{i}^{A}(t) \\
& =\sum_{i=1}^{n} \sum_{A \in \Lambda} \operatorname{sgn}\left(\boldsymbol{e}_{i}^{A}(t)\right)\left(-d_{i} \boldsymbol{e}_{i}^{A}(t)+\sum_{j=1}^{n} \sum_{B \in \Lambda} a_{i j}^{A . \bar{B}}\left(h_{j}^{B}\left(\boldsymbol{s}_{j}(t)\right)-h_{j}^{B}\left(\boldsymbol{r}_{j}(t)\right)\right)\right. \\
& +\sum_{j=1}^{n} \sum_{B \in \Lambda} b_{i j}^{A . \bar{B}}\left(h_{j}^{B}\left(\boldsymbol{s}_{j}\left(t-\tau_{j}(t)\right)\right)-h_{j}^{B}\left(\boldsymbol{r}_{j}\left(t-\tau_{j}(t)\right)\right)\right) \\
& \left.+\sum_{j=1}^{n} \sum_{B \in \Lambda} c_{i j}^{A . \bar{B}} \int_{t-\pi_{j}}^{t} \theta_{i j}(t-s)\left(h_{j}^{B}\left(\boldsymbol{s}_{j}(s)\right)-h_{j}^{B}\left(\boldsymbol{r}_{j}(s)\right)\right) d s+u_{i}^{A}(t)\right) \\
& =\sum_{i=1}^{n} \sum_{A \in \Lambda} \operatorname{sgn}\left(\boldsymbol{e}_{i}^{A}(t)\right)\left(-d_{i} \boldsymbol{e}_{i}^{A}(t)+\sum_{j=1}^{n} \sum_{B \in \Lambda} a_{i j}^{A} \cdot \bar{B}\left(h_{j}^{B}\left(\boldsymbol{s}_{j}(t)\right)-h_{j}^{B}\left(\boldsymbol{r}_{j}(t)\right)\right)\right. \\
& +\sum_{j=1}^{n} \sum_{B \in \Lambda} b_{i j}^{A \cdot \bar{B}}\left(h_{j}^{B}\left(\boldsymbol{s}_{j}\left(t-\tau_{j}(t)\right)\right)-h_{j}^{B}\left(\boldsymbol{r}_{j}\left(t-\tau_{j}(t)\right)\right)\right) \\
& +\sum_{j=1}^{n} \sum_{B \in \Lambda} c_{i j}^{A . \bar{B}} \int_{t-\pi_{j}}^{t} \theta_{i j}(t-s)\left(h_{j}^{B}\left(\boldsymbol{s}_{j}(s)\right)-h_{j}^{B}\left(\boldsymbol{r}_{j}(s)\right)\right) d s \\
& \left.-\alpha_{i} \boldsymbol{e}_{i}^{A}(t)-\beta_{i} \operatorname{sgn}\left(\left|\boldsymbol{e}_{i}^{A}(t)\right|\right)\right) \\
& \leq \sum_{i=1}^{n} \sum_{A \in \Lambda}-d_{i}\left|\boldsymbol{e}_{i}^{A}(t)\right|+\sum_{i=1}^{n} \sum_{j=1}^{n} \sum_{A \in \Lambda} \sum_{B \in \Lambda}\left|a_{i j}^{A} \cdot \bar{B}\right| l_{j}\left|\boldsymbol{e}_{j}^{A}(t)\right| \\
& +\sum_{i=1}^{n} \sum_{j=1}^{n} \sum_{A \in \Lambda} \sum_{B \in \Lambda}\left|b_{i j}^{A . \bar{B}}\right| l_{j}\left|\boldsymbol{e}_{j}^{A}\left(t-\tau_{j}(t)\right)\right| \\
& +\sum_{i=1}^{n} \sum_{j=1}^{n} \sum_{A \in \Lambda} \sum_{B \in \Lambda}\left|c_{i j}^{A . \bar{B}}\right| l_{j} \int_{t-\pi_{j}}^{t} \theta_{i j}(t-s)\left(\boldsymbol{e}_{j}(s)\right) d s \\
& -\sum_{i=1}^{n} \sum_{A \in \Lambda} \alpha_{i}\left|\boldsymbol{e}_{i}^{A}(t)\right|-\sum_{i=1}^{n} \sum_{A \in \Lambda} \beta_{i} \operatorname{sgn}\left(\left|\boldsymbol{e}_{i}^{A}(t)\right|\right) \\
& =\sum_{i=1}^{n} \sum_{A \in \Lambda}-\left(d_{i}+\alpha_{i}\right)\left|\boldsymbol{e}_{i}^{A}(t)\right|+\sum_{i=1}^{n} \sum_{j=1}^{n} \sum_{A \in \Lambda} \sum_{B \in \Lambda}\left|a_{i j}^{A} \cdot \bar{B}\right| l_{j}\left|\boldsymbol{e}_{j}^{A}(t)\right| \\
& +\sum_{i=1}^{n} \sum_{j=1}^{n} \sum_{A \in \Lambda} \sum_{B \in \Lambda}\left|b_{i j}^{A . \bar{B}}\right| l_{j}\left|\boldsymbol{e}_{j}^{A}\left(t-\tau_{j}(t)\right)\right| \\
& +\sum_{i=1}^{n} \sum_{j=1}^{n} \sum_{A \in \Lambda} \sum_{B \in \Lambda}\left|c_{i j}^{A . \bar{B}}\right| l_{j} \int_{t-\pi_{j}}^{t} \theta_{i j}(t-s)\left(\boldsymbol{e}_{j}(s)\right) d s-\sum_{i=1}^{n} \beta_{i} \lambda_{i},
\end{aligned}
$$

where $\lambda_{i}=1$ if $\left|\boldsymbol{e}_{i}^{A}(t)\right| \neq 0$, otherwise $\lambda_{i}=0$. It follows from Assumption 2 that 


$$
\begin{aligned}
\dot{\mathscr{V}}_{2}(t)= & \sum_{i=1}^{n} \sum_{j=1}^{n} \sum_{A \in \Lambda} \sum_{B \in \Lambda} \frac{1}{1-\mu_{j}}\left|b_{i j}^{A} \cdot \bar{B}\right| l_{j}\left|\boldsymbol{e}_{j}^{A}(t)\right| \\
& -\sum_{i=1}^{n} \sum_{j=1}^{n} \sum_{A \in \Lambda} \sum_{B \in \Lambda} \frac{1}{1-\mu_{j}}\left(1-\dot{\tau}_{j}(t)\right)\left|b_{i j}^{A . \bar{B}}\right| l_{j}\left|\boldsymbol{e}_{j}^{A}\left(t-\tau_{j}(t)\right)\right| \\
\leq & \sum_{i=1}^{n} \sum_{j=1}^{n} \sum_{A \in \Lambda} \sum_{B \in \Lambda} \frac{1}{1-\mu_{j}}\left|b_{i j}^{A . \bar{B}}\right| l_{j}\left|\boldsymbol{e}_{j}^{A}(t)\right| \\
& -\sum_{i=1}^{n} \sum_{j=1}^{n} \sum_{A \in \Lambda} \sum_{B \in \Lambda} \frac{1}{1-\mu_{j}}\left(1-\mu_{j}\right)\left|b_{i j}^{A} \cdot \bar{B}\right| l_{j}\left|\boldsymbol{e}_{j}^{A}\left(t-\tau_{j}(t)\right)\right| \\
= & \sum_{i=1}^{n} \sum_{j=1}^{n} \sum_{A \in \Lambda} \sum_{B \in \Lambda} \frac{1}{1-\mu_{j}}\left|b_{i j}^{A} \cdot \bar{B}\right| l_{j}\left|\boldsymbol{e}_{j}^{A}(t)\right|-\sum_{i=1}^{n} \sum_{j=1}^{n} \sum_{A \in \Lambda} \sum_{B \in \Lambda}\left|b_{i j}^{A} \cdot \bar{B}\right| l_{j}\left|\boldsymbol{e}_{j}^{A}\left(t-\tau_{j}(t)\right)\right|, \\
& \text { and } \dot{\mathscr{V}}_{3}(t)=\sum_{i=1}^{n} \sum_{j=1}^{n} \sum_{A \in \Lambda} \sum_{B \in \Lambda}\left|c_{i j}^{A} \cdot \bar{B}\right| l_{j} \int_{-\pi_{j}}^{0} \theta_{i j}(-s) \boldsymbol{e}_{i}^{A}(t) d s \\
& -\sum_{i=1}^{n} \sum_{j=1}^{n} \sum_{A \in \Lambda} \sum_{B \in \Lambda}\left|c_{i j}^{A . \bar{B}}\right| l_{j} \int_{-\pi_{j}}^{0} \theta_{i j}(-s) \boldsymbol{e}_{i}^{A}(t+s) d s \\
\leq & \sum_{i=1}^{n} \sum_{j=1}^{n} \sum_{A \in \Lambda} \sum_{B \in \Lambda}\left|c_{i j}^{A . \bar{B}}\right| l_{j} \tilde{\theta}_{i j} \boldsymbol{e}_{i}^{A}(t) \\
& -\sum_{i=1}^{n} \sum_{j=1}^{n} \sum_{A \in \Lambda} \sum_{B \in \Lambda}\left|c_{i j}^{A} \cdot \bar{B}\right| l_{j} \int_{t-\pi_{j}}^{t} \theta_{i j}(t-s) \boldsymbol{e}_{i}^{A}(s) d s .
\end{aligned}
$$

Combining (20)-(22), we obtain

$$
\begin{aligned}
\dot{\mathscr{V}}(t) \leq & \sum_{i=1}^{n} \sum_{A \in \Lambda}-\left(d_{i}+\alpha_{i}\right)\left|\boldsymbol{e}_{i}^{A}(t)\right|+\sum_{i=1}^{n} \sum_{j=1}^{n} \sum_{A \in \Lambda} \sum_{B \in \Lambda}\left|a_{i j}^{A} \cdot \bar{B}\right| l_{j}\left|\boldsymbol{e}_{j}^{A}(t)\right| \\
& +\sum_{i=1}^{n} \sum_{j=1}^{n} \sum_{A \in \Lambda} \sum_{B \in \Lambda}\left|b_{i j}^{A} \cdot \bar{B}\right| l_{j}\left|\boldsymbol{e}_{j}^{A}\left(t-\tau_{j}(t)\right)\right| \\
& +\sum_{i=1}^{n} \sum_{j=1}^{n} \sum_{A \in \Lambda} \sum_{B \in \Lambda}\left|c_{i j}^{A . \bar{B}}\right| l_{j} \int_{t-\pi_{j}}^{t} \theta_{i j}(t-s)\left(\boldsymbol{e}_{j}(s)\right) d s-\sum_{i=1}^{n} \sum_{A \in \Lambda} \alpha_{i}\left|\boldsymbol{e}_{i}^{A}(t)\right| \\
& -\sum_{i=1}^{n} \beta_{i} \lambda_{i}+\sum_{i=1}^{n} \sum_{j=1}^{n} \sum_{A \in \Lambda} \sum_{B \in \Lambda} \frac{1}{1-\mu_{j}}\left|b_{i j}^{A . \bar{B}}\right| l_{j}\left|\boldsymbol{e}_{j}^{A}(t)\right| \\
& -\sum_{i=1}^{n} \sum_{j=1}^{n} \sum_{A \in \Lambda} \sum_{B \in \Lambda}\left|b_{i j}^{A . \bar{B}}\right| l_{j}\left|\boldsymbol{e}_{j}^{A}\left(t-\tau_{j}(t)\right)\right|+\sum_{i=1}^{n} \sum_{j=1}^{n} \sum_{A \in \Lambda} \sum_{B \in \Lambda}\left|c_{i j}^{A . \bar{B}}\right| l_{j} \tilde{\theta}_{i j} \boldsymbol{e}_{i}^{A}(t) \\
& -\sum_{i=1}^{n} \sum_{j=1}^{n} \sum_{A \in \Lambda} \sum_{B \in \Lambda}\left|c_{i j}^{A} \cdot \bar{B}\right| l_{j} \int_{t-\pi_{j}}^{t} \theta_{i j}(t-s) \boldsymbol{e}_{i}^{A}(s) d s \\
= & \sum_{i=1}^{n} \sum_{A \in \Lambda}\left(-d_{i}-\alpha_{i}+\sum_{j=1}^{n} \sum_{B \in \Lambda} l_{j}\left|a_{i j}^{A} \cdot \bar{B}\right|+\frac{1}{1-\mu_{j}} \sum_{j=1}^{n} \sum_{B \in \Lambda} l_{j}\left|b_{i j}^{A . \bar{B}}\right|\right. \\
& \left.+\sum_{j=1}^{n} \sum_{B \in \Lambda} l_{j}\left|c_{i j}^{A . \bar{B}}\right| \tilde{\theta}_{i j}\right)\left|\boldsymbol{e}_{i}^{A}(t)\right|-\sum_{i=1}^{n} \beta_{i} \lambda_{i} .
\end{aligned}
$$


Substituting condition (14) into (23), we obtain:

$$
\dot{\mathscr{V}}(t) \leq-\sum_{i=1}^{n} \beta_{i} \lambda_{i} \leq 0 .
$$

Using $\mathscr{V}(t)$ in (15), which is positive definite, we have nonnegative constant $\mathscr{V}^{*}$ and $t_{1} \in(0,+\infty)$ such as

$$
\lim _{t \rightarrow t_{1}}=\mathscr{V}^{*} \text { and } \mathscr{V}(t) \equiv \mathscr{V}^{*}, \forall t \geq t_{1}
$$

Next, we will demonstrate that

$$
\left\|\boldsymbol{e}^{A}\left(t_{1}\right)\right\|_{1}=0 \text { and }\left\|\boldsymbol{e}^{A}\left(t_{1}\right)\right\|_{1} \equiv 0, \forall t \geq t_{1} .
$$

Firstly, we prove that $\left\|\boldsymbol{e}^{A}\left(t_{1}\right)\right\|_{1}=0$; otherwise $\left\|\boldsymbol{e}^{A}\left(t_{1}\right)\right\|_{1}>0$. As such, there is a the small constant $\epsilon>0$ such as $\left\|\boldsymbol{e}^{A}\left(t_{1}\right)\right\|_{1}>0$ for all $t \in\left[t_{1}, t_{1}+\epsilon\right]$, so there exists at least one $k_{0} \in N$ such that $\left|\boldsymbol{e}_{k_{0}}^{A}(t)\right|>0$ for $t \in\left[t_{1}, t_{1}+\epsilon\right]$, leading to $\dot{\mathscr{V}}(t) \leq-\beta_{k_{0}}<0$ that holds for all $t \in\left[t_{1}, t_{1}+\epsilon\right]$. This presents a contradiction to (25).

Secondly, we prove $\left\|\boldsymbol{e}^{A}(t)\right\|_{1} \equiv 0$ for $t \geq t_{1}$. Using contradiction, suppose, without loss of generality, that there exists at least one $k_{0} \in N$ and $t_{2}>t_{1}$ such that $\left|\boldsymbol{e}_{k_{0}}^{A}\left(t_{2}\right)\right|_{1}>0$. Let $t_{s}=\sup \left\{t \in\left[t_{1}, t_{2}\right]:\left\|\boldsymbol{e}^{A}(t)\right\|_{1}=0\right\}$, we have $t_{s}<t_{2},\left\|\boldsymbol{e}^{A}\left(t_{s}\right)\right\|_{1}=0$ and $\left|\boldsymbol{e}_{k_{0}}^{A}(t)\right|_{1}>0$ for all $t \in\left(t_{s}, t_{2}\right]$. In addition, there exists $t_{3} \in\left(t_{s}, t_{2}\right]$ such that $\left|\boldsymbol{e}_{k_{0}}^{A}(t)\right|$ is monotonously increasing to the interval $\left[t_{s}, t_{3}\right]$; therefore, $\mathscr{V}(t)$ is also monotonously increasing pertaining to $\left[t_{s}, t_{3}\right]$, i.e., $\dot{\mathscr{V}}(t)>0$ for $t \in\left(t_{s}, t_{3}\right]$. Moreover, according to the first part of the discussion, we can obtain $\dot{\mathscr{V}}(t) \leq-\beta_{k_{0}}<0$ holds for all $t \in\left[t_{s}, t_{3}\right]$; and this presents a contradiction. As a result, $\left\|\boldsymbol{e}^{A}(t)\right\|_{1} \equiv 0$ for $t \geq t_{1}$.

In summary, the conditions in (26) hold. Referring to Definition (2), the NN models (7) and (8) are synchronize with the NN models (9) and (10) in finite time under controller (13). This completes the proof.

Corollary 5. Consider $c_{i j}=0, i, j \in N$. Given that Assumptions 1 and 2 are satisfied and

$$
d_{i}+\alpha_{i}-\left(\sum_{i=1}^{n} \sum_{j=1}^{n} \sum_{A \in \Lambda} \sum_{B \in \Lambda} l_{j}\left|a_{i j}^{A} \cdot \bar{B}\right|+\sum_{i=1}^{n} \sum_{j=1}^{n} \sum_{A \in \Lambda} \sum_{B \in \Lambda} \frac{1}{1-\mu_{j}} l_{j}\left|b_{i j}^{A . \bar{B}}\right|\right)>0, i, j \in N,
$$

then the Clifford-valued NN models (7) and (8) are synchronized with the NN models (9) and (10) in finite-time under controller (13).

Corollary 6. Consider that $b_{i j}=c_{i j}=0, i, j \in N$. Given Assumption 2 is satisfied and

$$
d_{i}+\alpha_{i}-\sum_{i=1}^{n} \sum_{j=1}^{n} \sum_{A \in \Lambda} \sum_{B \in \Lambda} l_{j}\left|a_{i j}^{A} \cdot \bar{B}\right|>0, i, j \in N,
$$

then the Clifford-valued NN models (7) and (8) are synchronized with the NN models (9) and (10) in finite-time under controller (13).

Remark 7. It is well known that the multiplication of the Clifford numbers does not comply with the commutative law, which complicates the investigation of the Clifford NNs dynamics. Although the known results regarding Clifford-valued NNs are still limited, we know that the decomposition approach is very efficient to solve the problem of non commutativity of the multiplication of the Clifford numbers. Thus, the use of decomposition to analyze Clifford-valued NNs is highly meaningful. Recently, most of the results are obtained through the decomposition method [28,29,48-50]. 
Remark 8. In Theorem (4) by decomposing the original n-dimensional Clifford-valued system into a multidimensional real-valued system, several sufficient conditions are derived to show the considered system model is finite-time synchronization, but our result is really about Clifford-valued systems themselves.

Remark 9. It should be pointed out that Clifford-valued neural networks aim to explore new capabilities and improve accuracy in order to solve problems that cannot be solved by real-, complexand quaternion-valued NNs. For instance, the results of finite-time synchronization of complexvalued NNs [44,45] can then be summarized as a special case of the results of this paper.

Remark 10. Many important results have recently been published concerning the various dynamics of Clifford valued NNs [28-35,48-50]. Specific examples includes $S^{p}$-Almost periodic solutions [30], globally asymptotic almost automorphic synchronization [31], global exponential stability of pseudo almost periodic solution [32], global stability analysis [50], and so on. However, any work on the topic of finite-synchronization of Clifford-valued NNs with time-varying delays has not yet been reported. As a result, in order to fill such gaps, we (for the first time) derived new sufficient conditions to ensure the finite-time synchronization of Clifford-valued NNs models with time delays. Therefore, this paper's main results are new and different compared with those in the existing literature.

\section{Numerical Examples}

We present a numerical example to demonstrate the feasibility and effectiveness of the main results established in Section 3.

Example 1. For $m=2$ and $n=2$, the following two-neuron drive model (1) is considered:

$$
\begin{aligned}
\dot{\boldsymbol{r}}_{i}(t)= & -d_{i} \boldsymbol{r}_{i}(t)+\sum_{j=1}^{2} a_{i j} h_{j}\left(\boldsymbol{\gamma}_{j}(t)\right)+\sum_{j=1}^{2} b_{i j} h_{j}\left(\boldsymbol{r}_{j}\left(t-\tau_{j}(t)\right)\right) \\
& +\sum_{j=1}^{2} c_{i j} \int_{t-\pi_{j}}^{t} \theta_{i j}(t-s) h_{j}\left(\boldsymbol{r}_{j}(s)\right) d s+k_{i}, t \geq 0 .
\end{aligned}
$$

The corresponding response model (3) is:

$$
\begin{aligned}
\dot{\boldsymbol{s}}_{i}(t)= & -d_{i} \boldsymbol{s}_{i}(t)+\sum_{j=1}^{2} a_{i j} h_{j}\left(\boldsymbol{s}_{j}(t)\right)+\sum_{j=1}^{2} b_{i j} h_{j}\left(\boldsymbol{s}_{j}\left(t-\tau_{j}(t)\right)\right) \\
& +\sum_{j=1}^{2} c_{i j} \int_{t-\pi_{j}}^{t} \theta_{i j}(t-s) h_{j}\left(\boldsymbol{s}_{j}(s)\right) d s+k_{i}+u_{i}(t), t \geq 0 .
\end{aligned}
$$

The multiplication generators are: $e_{1}^{2}=e_{2}^{2}=e_{12}^{2}=e_{1} e_{2} e_{12}=-1, e_{1} e_{2}=-e_{2} e_{1}=e_{12}$, $e_{1} e_{12}=-e_{12} e_{1}=-e_{2}, e_{2} e_{12}=-e_{12} e_{2}=e_{1}, \boldsymbol{r}_{1}=\boldsymbol{r}_{1}^{0} e_{0}+\boldsymbol{r}_{1}^{1} e_{1}+\boldsymbol{r}_{1}^{2} e_{2}+\boldsymbol{r}_{1}^{12} e_{12}, \boldsymbol{r}_{2}=\boldsymbol{r}_{2}^{0} e_{0}+$ $\boldsymbol{r}_{2}^{1} e_{1}+\boldsymbol{r}_{2}^{2} e_{2}+\boldsymbol{r}_{2}^{12} e_{12}, \boldsymbol{s}_{1}=\boldsymbol{s}_{1}^{0} e_{0}+\boldsymbol{s}_{1}^{1} e_{1}+\boldsymbol{s}_{1}^{2} e_{2}+\boldsymbol{s}_{1}^{12} e_{12}, \boldsymbol{s}_{2}=\boldsymbol{s}_{2}^{0} e_{0}+\boldsymbol{s}_{2}^{1} e_{1}+\boldsymbol{s}_{2}^{2} e_{2}+\boldsymbol{s}_{2}^{12} e_{12}$. 
Furthermore, we take

$$
\begin{aligned}
& d_{1}=d_{2}=5 \text {, } \\
& a_{11}=0.5 e_{0}+0.1 e_{1} \text {, } \\
& a_{12}=0.1 e_{0}+0.2 e_{2}+0.6 e_{12} \text {, } \\
& a_{21}=0.5 e_{0}-0.1 e_{1}+0.3 e_{2} \text {, } \\
& a_{22}=0.3 e_{0}+0.1 e_{1}+0.5 e_{12} \text {, } \\
& b_{11}=0.1 e_{0}+0.2 e_{1}+0.5 e_{2} \text {, } \\
& b_{12}=0.3 e_{0}+0.1 e_{2}+0.4 e_{12} \text {, } \\
& b_{21}=0.6 e_{0}-0.2 e_{1}+0.3 e_{2} \text {, } \\
& b_{22}=0.4 e_{0}+0.1 e_{12} \text {, } \\
& c_{11}=0.1 e_{0}+0.2 e_{2}+0.6 e_{12} \text {, } \\
& c_{12}=0.3 e_{0}+0.1 e_{1}+0.5 e_{12} \text {, } \\
& c_{21}=0.1 e_{0}+0.2 e_{1}+0.5 e_{2} \text {, } \\
& c_{22}=0.6 e_{0}-0.2 e_{1}+0.3 e_{2} \text {, } \\
& k_{1}=0.1 e_{0}-0.2 e_{1}+0.2 e_{2}+0.1 e_{12} \text {, } \\
& k_{2}=-0.2 e_{0}+0.2 e_{1}+0.1 e_{2}+0.1 e_{12} \text {, } \\
& g_{1}\left(\boldsymbol{e}_{1}\right)=\frac{1-e^{-\boldsymbol{e}_{1}^{0}}}{1+e^{-\boldsymbol{e}_{1}^{0}}} e_{0}+\frac{1}{1+e^{-\boldsymbol{e}_{1}^{1}}} e_{1}+\frac{1-e^{-\boldsymbol{e}_{1}^{2}}}{1+e^{-\boldsymbol{e}_{1}^{2}}} e_{2}+\frac{1}{1+e^{-\boldsymbol{e}_{1}^{12}}} e_{12}, \\
& g_{2}\left(\boldsymbol{e}_{2}\right)=\frac{1-e^{-\boldsymbol{e}_{2}^{0}}}{1+e^{-\boldsymbol{e}_{2}^{0}}} e_{0}+\frac{1}{1+e^{-\boldsymbol{e}_{2}^{1}}} e_{1}+\frac{1-e^{-\boldsymbol{e}_{2}^{2}}}{1+e^{-\boldsymbol{e}_{2}^{2}}} e_{2}+\frac{1}{1+e^{-\boldsymbol{e}_{2}^{12}}} e_{12} .
\end{aligned}
$$

in which $\boldsymbol{e}_{i}(t)=\boldsymbol{s}_{i}(t)-\boldsymbol{r}_{i}(t)$ and $\boldsymbol{e}_{i}^{A}(t)=\boldsymbol{s}_{i}^{A}(t)-\boldsymbol{r}_{i}^{A}(t), i=1,2$. Consider the following time-varying delays $\tau_{1}(t)=\tau_{2}(t)=0.6|\cos (t)|+0.4, \pi_{1}=\pi_{2}=0.8, \theta_{i j}(t)=e^{-0.5 t}$, $i, j \in N$. It is obvious to obtain that $\tau_{1}=\tau_{2}=1, \mu_{j}=0.6<1$ and $\tilde{\theta}_{i j}=2(i, j \in N)$. Furthermore, the activation function satisfies Assumption 2 with $l_{1}=l_{2}=0.5$ by selecting $\alpha_{1}=2.5, \alpha_{2}=2.6, \beta_{1}=3.5$ and $\beta_{2}=3.8$.

In addition, it is easy to obtain $a_{11}^{A . \bar{B}}=0.6, a_{12}^{A . \bar{B}}=0.9, a_{21}^{A . \bar{B}}=0.7, a_{22}^{A . \bar{B}}=0.9$, $b_{11}^{A . \bar{B}}=0.8, b_{12}^{A . \bar{B}}=0.8, b_{21}^{A . \bar{B}}=0.7, b_{22}^{A . \bar{B}}=0.5, c_{11}^{A . \bar{B}}=0.9, c_{12}^{A . \bar{B}}=0.9, c_{21}^{A . \bar{B}}=0.8, c_{22}^{A . \bar{B}}=0.7$. We consider the initial conditions of the drive-response models (1) $-(4)$ as follows: $\varphi_{1}(t)=$ $-1.5 e_{0}+1.2 e_{1}+0.9 e_{2}+0.5 e_{12}$ for $t \in[-1,0], \varphi_{2}(t)=1.6 e_{0}-3.5 e_{1}+2.2 e_{2}-0.9 e_{12}$ for $t \in[-1,0], \phi_{1}(t)=2.5 e_{0}-e_{1}+1.5 e_{2}-1.2 e_{12}$ for $t \in[-1,0]$, and $\phi_{2}(t)=-2.6 e_{0}-2.6 e_{1}-$ $2.2 e_{2}+0.8 e_{12}$ for $t \in[-1,0]$.

By simple computation, we have

$$
\begin{aligned}
d_{i}+\alpha_{i}- & \left(\sum_{i=1}^{n} \sum_{j=1}^{n} \sum_{A \in \Lambda} \sum_{B \in \Lambda} l_{j}\left|a_{i j}^{A . \bar{B}}\right|+\sum_{i=1}^{n} \sum_{j=1}^{n} \sum_{A \in \Lambda} \sum_{B \in \Lambda} \frac{1}{1-\mu_{j}} l_{j}\left|b_{i j}^{A . \bar{B}}\right|\right. \\
& \left.+\sum_{i=1}^{n} \sum_{j=1}^{n} \sum_{A \in \Lambda} \sum_{B \in \Lambda} l_{j}\left|c_{i j}^{A . \bar{B}}\right| \tilde{\theta}_{i j}\right)=3.95>0, \\
d_{i}+\alpha_{i}- & \left(\sum_{i=1}^{n} \sum_{j=1}^{n} \sum_{A \in \Lambda} \sum_{B \in \Lambda} l_{j}\left|a_{i j}^{A . \bar{B}}\right|+\sum_{i=1}^{n} \sum_{j=1}^{n} \sum_{A \in \Lambda} \sum_{B \in \Lambda} \frac{1}{1-\mu_{j}} l_{j}\left|b_{i j}^{A . \bar{B}}\right|\right. \\
& \left.+\sum_{i=1}^{n} \sum_{j=1}^{n} \sum_{A \in \Lambda} \sum_{B \in \Lambda} l_{j}\left|c_{i j}^{A . \bar{B}}\right| \tilde{\theta}_{i j}\right)=3.45>0 .
\end{aligned}
$$

To sum up, we have shown that all conditions in Theorem (4) are satisfied. The driveresponse models (1) and (3) can achieve synchronization in finite-time with controller (13). The following is a detailed explanation of Figures 1-12. Figures 1, 2, 4, 5, 7, 8, 10 and 11, respectively, show the time responses of the states of the drive-response models (1) and (3). 
Besides, Figures 3, 6, 9 and 12 disclose the time responses of the states of the error systems (11). From Figures 3, 6, 9 and 12, it can be seen that model (7) synchronize with model (9) in finite-time through the controller (13) with the given initial values.

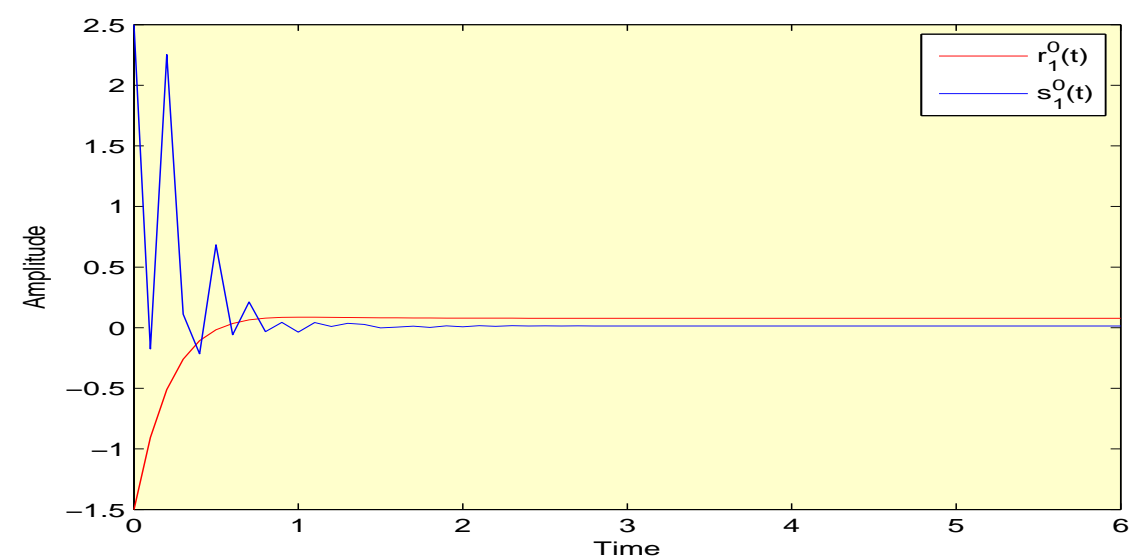

Figure 1. Time responses of state variables $\boldsymbol{r}_{1}^{0}(t), \boldsymbol{s}_{1}^{0}(t)$ of NN models (1) and (3).

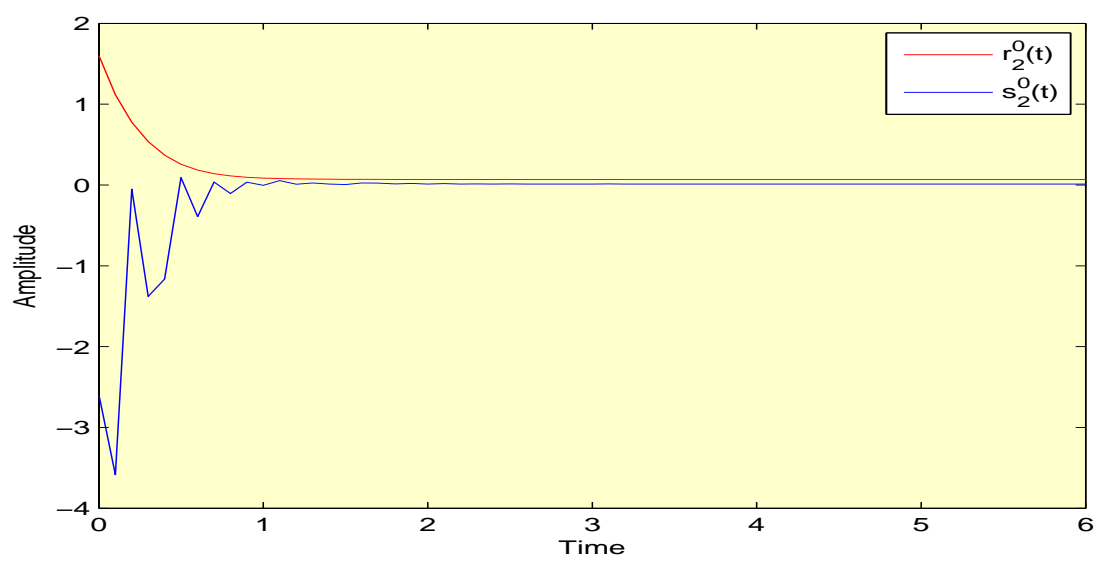

Figure 2. Time responses of state variables $\boldsymbol{r}_{2}^{0}(t), \boldsymbol{s}_{2}^{0}(t)$ of NN models (1) and (3).

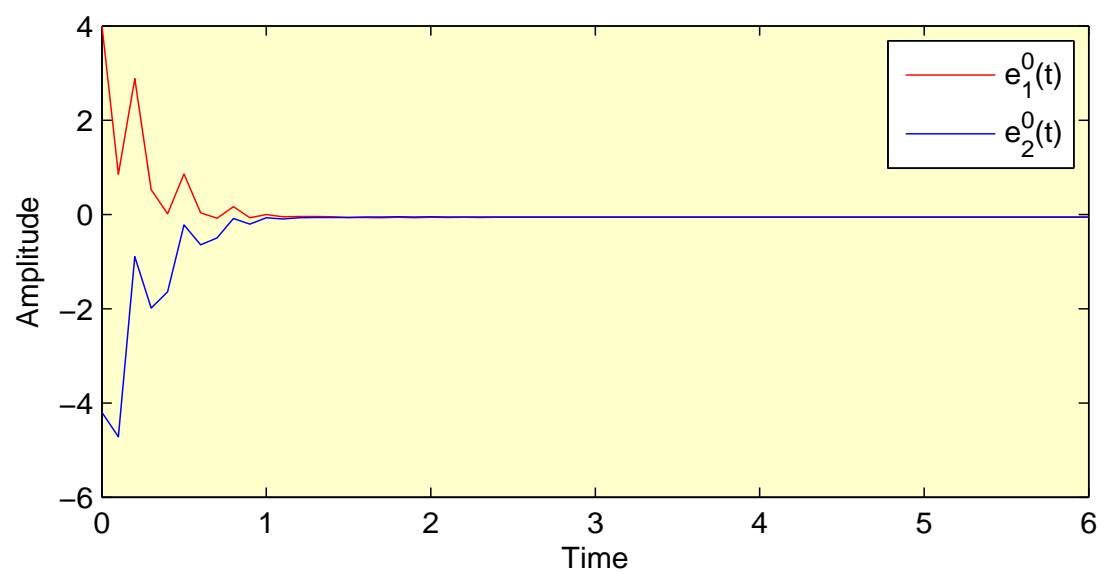

Figure 3. Synchronization curves of state variables $\boldsymbol{e}_{1}^{0}(t), \boldsymbol{e}_{2}^{0}(t)$ of NN model (11) under controller (13). 


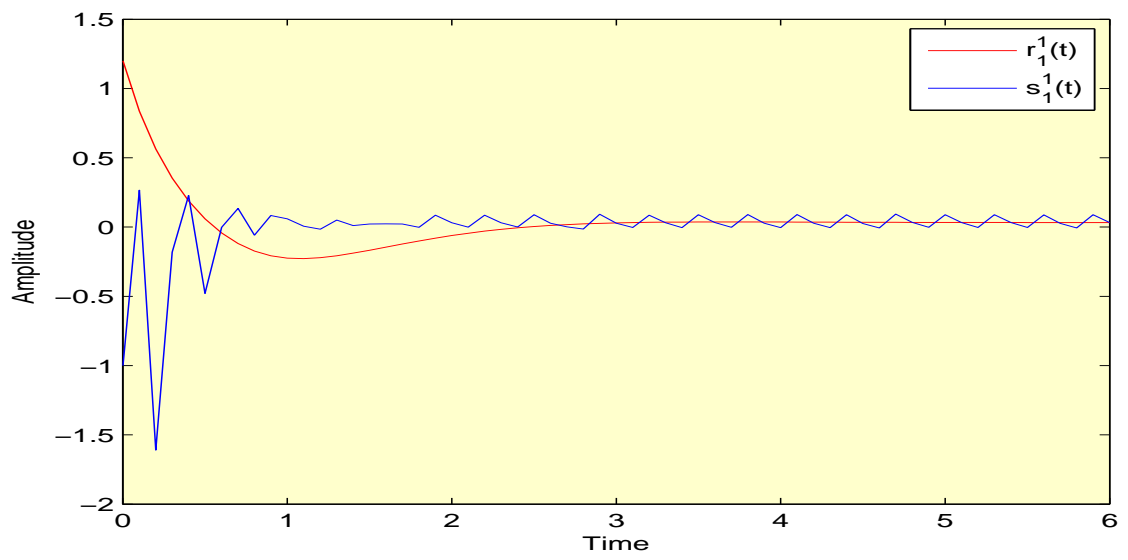

Figure 4. Time responses of state variables $\boldsymbol{r}_{1}^{1}(t), \boldsymbol{s}_{1}^{1}(t)$ of NN models (1) and (3).

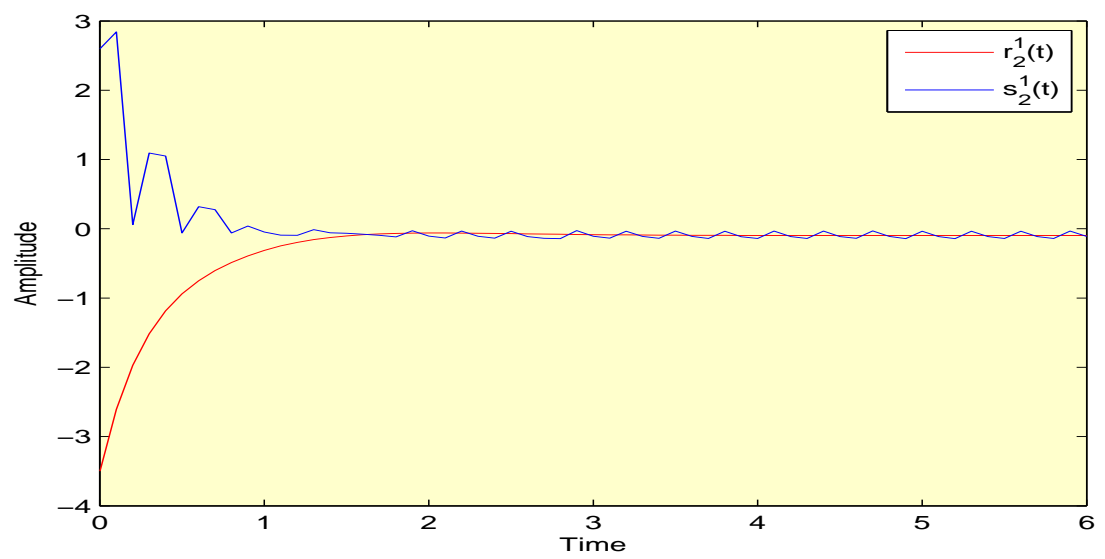

Figure 5. Time responses of state variables $\boldsymbol{r}_{2}^{1}(t), \boldsymbol{s}_{2}^{1}(t)$ of NN models (1) and (3).

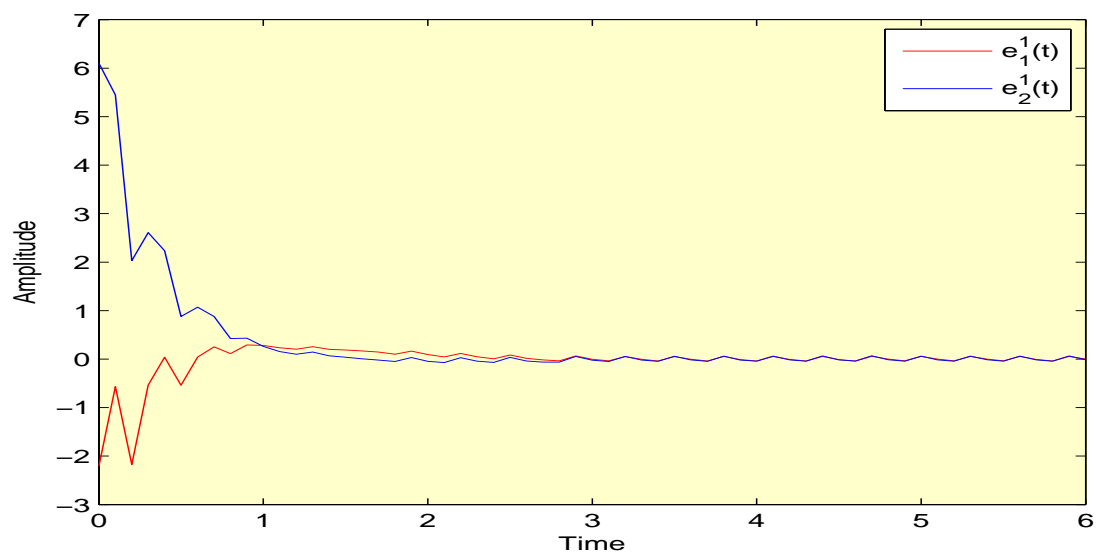

Figure 6. Synchronization curves of state variables $\boldsymbol{e}_{1}^{1}(t), \boldsymbol{e}_{2}^{1}(t)$ of NN model (11) under controller (13). 


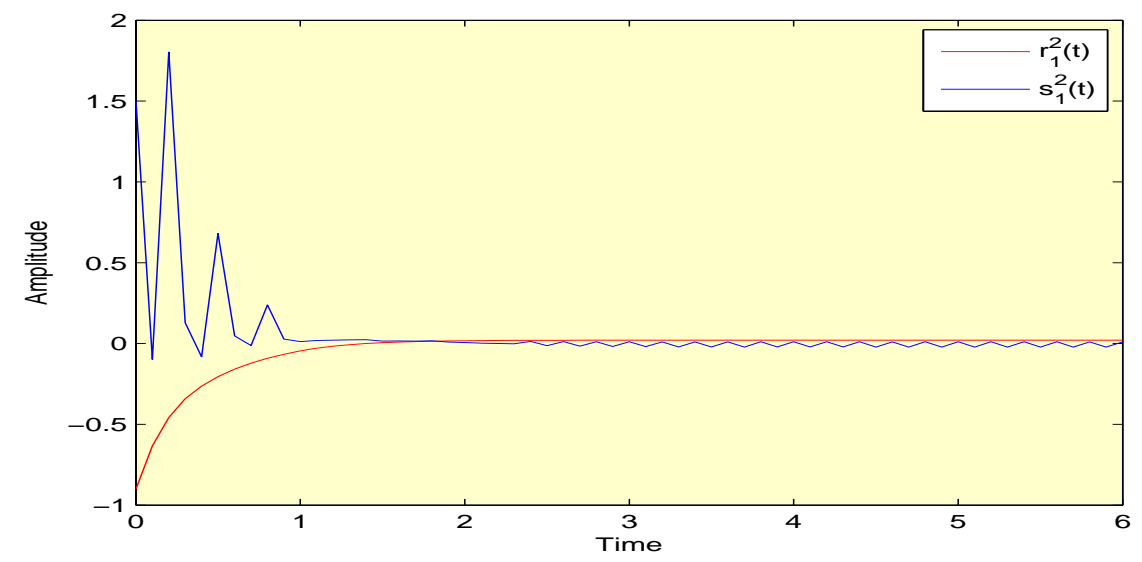

Figure 7. Time responses of state variables $\boldsymbol{r}_{1}^{2}(t), \boldsymbol{s}_{1}^{2}(t)$ of NN models (1) and (3).

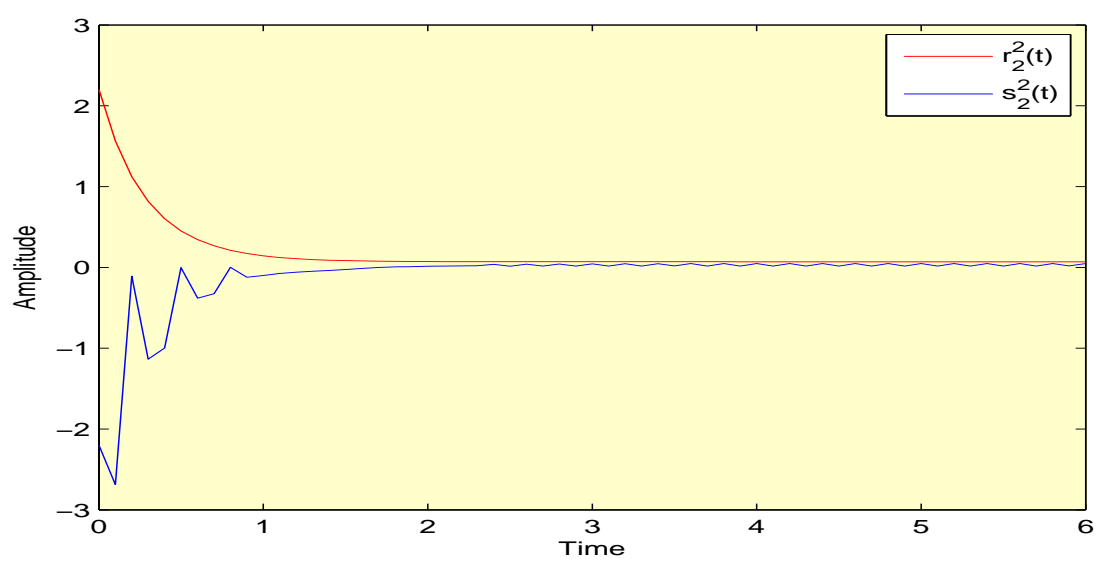

Figure 8. Time responses of state variables $\boldsymbol{r}_{2}^{2}(t), \boldsymbol{s}_{2}^{2}(t)$ of NN models (1) and (3).

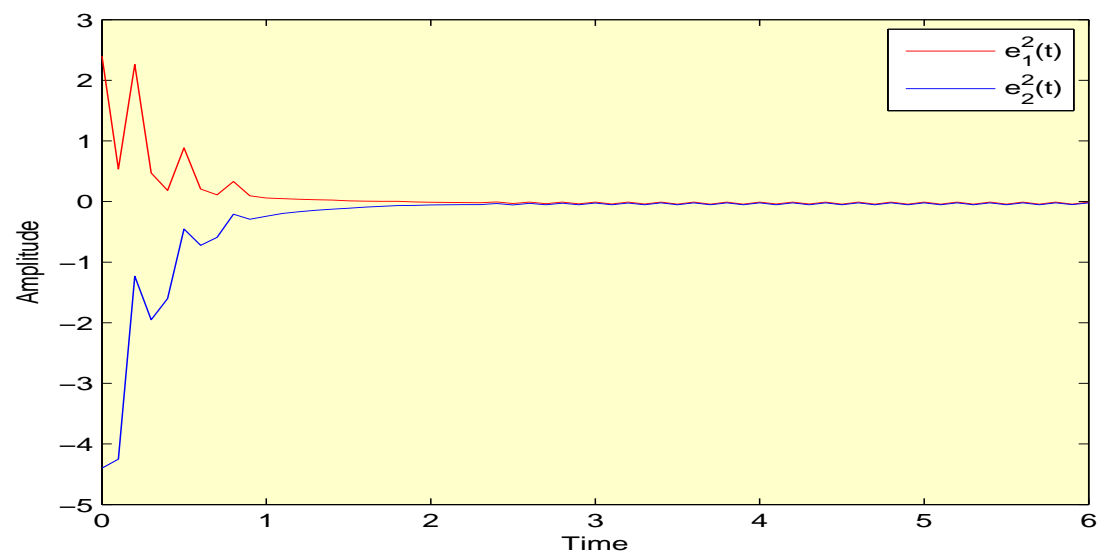

Figure 9. Synchronization curves of state variables $\boldsymbol{e}_{1}^{2}(t), \boldsymbol{e}_{2}^{2}(t)$ of NN model (11) under controller (13). 


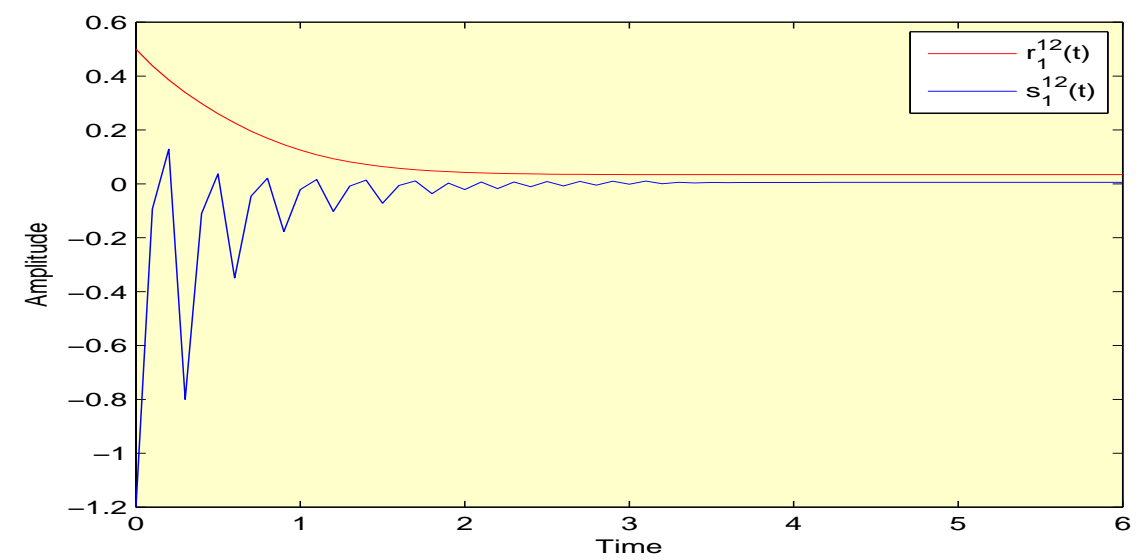

Figure 10. Time responses of state variables $\boldsymbol{r}_{1}^{12}(t), \boldsymbol{s}_{1}^{12}(t)$ of NN models (1) and (3).

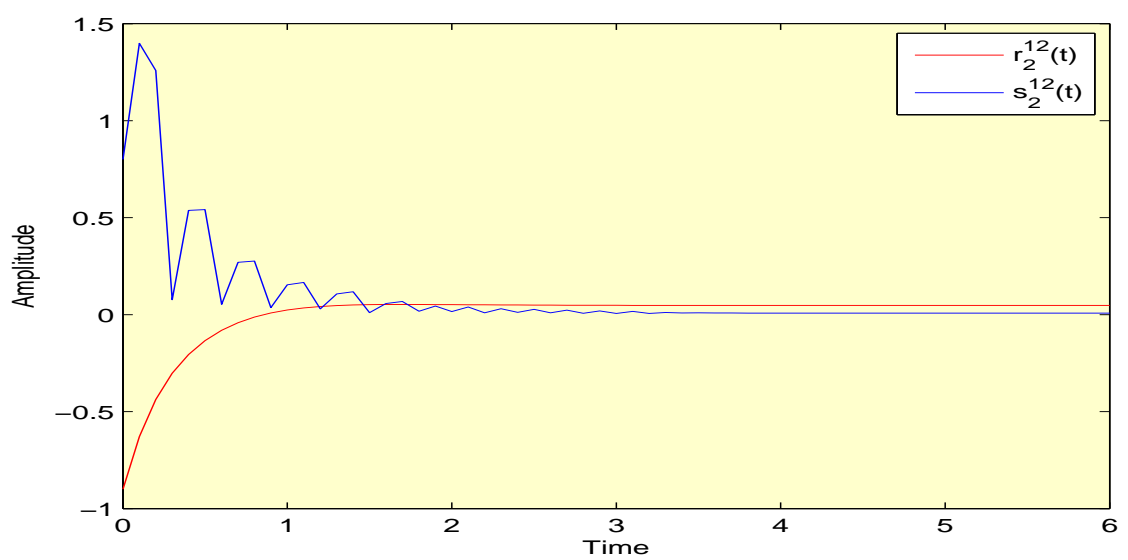

Figure 11. Time responses of state variables $\boldsymbol{r}_{2}^{12}(t), \boldsymbol{s}_{2}^{12}(t)$ of NN models (1) and (3).

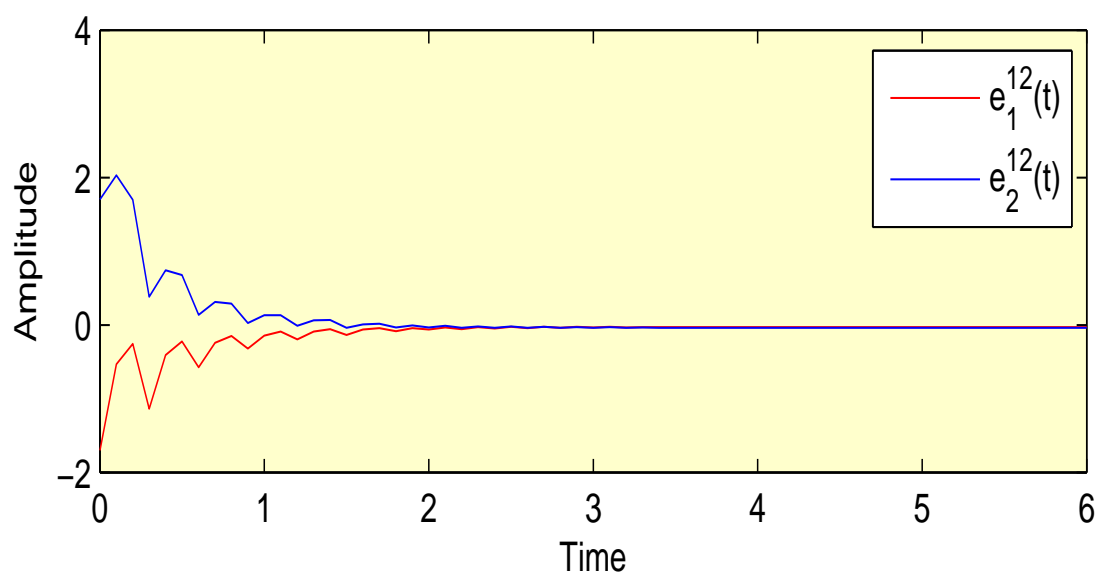

Figure 12. Synchronization curves of state variables $\boldsymbol{e}_{1}^{12}(t), \boldsymbol{e}_{2}^{12}(t)$ of NN model (11) under controller (13).

\section{Conclusions}

In this article, we have investigated the finite-time synchronization of Clifford-valued NN models with finite-time distributed delays. To address the problem of non-commutativity of multiplication of Clifford numbers, the original Clifford-valued drive and response models are firstly decomposed into the corresponding real-valued drive and response counterparts. Moreover, suitable controller has been constructed to examine the synchronization problem associated with the finite-time error models. On the basis of a new Lyapunov-Krasovskii 
functional and some new computational techniques, finite-time synchronization criteria have been derived for the obtained real-valued drive-response models. In addition, we have presented a numerical simulation to illustrate the usefulness of the main results. The results obtained in this paper can be further extended to other complex systems. Accordingly, we plan to extend our results to more general Clifford-valued NN models, such as Clifford-valued inertial NNs, Clifford-valued high-order Hopfield NNs, and fuzzy Clifford-valued NNs. Moreover, we will focus on the problem of global stabilization analysis of Clifford-valued NN models with the help of various controller approaches.

Author Contributions: Funding acquisition, P.S.-a.; Conceptualization, G.R.; Software, G.R. and P.S.-a.; Formal analysis, G.R.; Methodology, G.R. and P.S.-a.; Supervision, G.R., P.H., R.S. and C.P.L.; Writing-original draft, G.R.; Validation, G.R. and P.S.; Writing-review and editing, G.R. and P.S.-a. All authors have read and agreed to the published version of the manuscript.

Funding: This research was supported by Suranaree University of Technology.

Institutional Review Board Statement: Not applicable.

Informed Consent Statement: Not applicable.

Data Availability Statement: No data were used to support this study.

Conflicts of Interest: The authors declare that they have no conflict of interest.

\section{References}

1. Cao, J.; Wang, J. Global asymptotic stability of a general class of recurrent neural networks with time-varying delays. IEEE Trans. Circuits Syst. I 2003, 50, 34-44.

2. Cao, J.; Wang, J. Global asymptotic and robust stability of recurrent neural networks with time delays. IEEE Trans. Circuits Syst. I 2005, 52, 417-426.

3. Cao, J.; Yuan, K.; Li, H. Global asymptotical stability of recurrent neural networks with multiple discrete delays and distributed delays. IEEE Trans. Neural Netw. 2006, 17, 1646-1651. [PubMed]

4. Chen, J.; Zhang, X.M.; Park, J.H.; Xu, S. Improved stability criteria for delayed neural networks using a quadratic function negative-definiteness approach. IEEE Trans. Neural Netw. Learn. Syst. 2020. [CrossRef] [PubMed]

5. Lu, C.; Zhang, X.M.; Wu, M.; Han, Q.L.; He, Y. Receding horizon synchronization of delayed neural networks using a novel inequality on quadratic polynomial functions. IEEE Trans. Syst. Man Cyber. Syst. 2019. [CrossRef]

6. Zhang, X.M.; Han, Q.L.; Ge, X.; Ding, D. An overview of recent developments in Lyapunov-Krasovskii functionals and stability criteria for recurrent neural networks with time-varying delays. Neurocomputing 2018, 313, 392-401. [CrossRef]

7. Hirose, A. Complex-Valued Neural Networks: Theories and Applications; World Scientific: Singapore, 2003.

8. Isokawa, T.; Nishimura, H.; Kamiura, N.; Matsui, N. Associative memory in quaternionic Hopfield neural network. Int. J. Neural Syst. 2008, 18, 135-145. [CrossRef]

9. Matsui, N.; Isokawa, T.; Kusamichi, H.; Peper, F.; Nishimura, H. Quaternion neural network with geometrical operators. J. Intell. Fuzzy Syst. 2004, 15, 149-164.

10. Mandic, D.P.; Jahanchahi, C.; Took, C.C. A quaternion gradient operator and its applications. IEEE Signal Proc. Lett. 2011, 18, 47-50. [CrossRef]

11. Nitta, T. Solving the XOR problem and the detection of symmetry using a single complex-valued neuron. Neural Netw. 2003, 16, 1101-1105. [CrossRef]

12. Zhang, Z., Liu, X., GuoR., Lin, C. Finite-time stability for delayed complex-valued BAM neural networks. Neural Process. Lett. 2018, 48, 179-193. [CrossRef]

13. Zhang, Z.; Liu, X.; Chen, J.; Guo, R.; Zhou, S. Further stability analysis for delayed complex-valued recurrent neural networks. Neurocomputing 2017, 251, 81-89. [CrossRef]

14. Zhang, Z.; Liu, X.; Zhou, D.; Lin, C.; Chen, J.; Wang, H. Finite-time stabilizability and instabilizability for complex-valued memristive neural networks with time delays. IEEE Trans. Syst. Man Cybern. Syst. 2018, 48, 2371-2382. [CrossRef]

15. Samidurai, R.; Sriraman, R.; Zhu, S. Leakage delay-dependent stability analysis for complex-valued neural networks with discrete and distributed time-varying delays. Neurocomputing 2019, 338, 262-273. [CrossRef]

16. Li, Y.; Meng, X. Almost automorphic solutions for quaternion-valued Hopfield neural networks with mixed time-varying delays and leakage delays. J. Syst. Sci. Complex. 2020, 33, 100-121. [CrossRef]

17. Tu, Z.; Zhao, Y.; Ding, N.; Feng, Y.; Zhang, W. Stability analysis of quaternion-valued neural networks with both discrete and distributed delays. Appl. Math. Comput. 2019, 343, 342-353. [CrossRef]

18. Shu, H.; Song, Q.; Liu, Y.; Zhao, Z.; Alsaadi, F.E. Global $\mu$-stability of quaternion-valued neural networks with non-differentiable time-varying delays. Neurocomputing 2017, 247, 202-212. [CrossRef] 
19. Tan, M.; Liu, Y.; Xu, D. Multistability analysis of delayed quaternion-valued neural networks with nonmonotonic piecewise nonlinear activation functions. Appl. Math. Comput. 2019, 341, 229-255. [CrossRef]

20. Pearson, J.K.; Bisset, D.L. Neural networks in the Clifford domain. In Proceedings of the Proceedings of 1994 IEEE International Conference on Neural Networks (ICNN'94), Orlando, FL, USA, 28 June-2 July 1994.

21. Pearson, J.K.; Bisset, D.L. Back Propagation in a Clifford Algebra; ICANN: Brighton, UK, 1992.

22. Buchholz, S. A Theory of Neural Computation with Clifford Algebras. Ph.D. Thesis, University of Kiel, Kiel, Germany, 2005.

23. Buchholz, S.; Sommer, G. On Clifford neurons and Clifford multi-layer perceptrons. Neural Netw. 2008, 21, 925-935. [CrossRef] [PubMed]

24. Kuroe, Y.; Models of Clifford recurrent neural networks and their dynamics. In Proceedings of the 2011 International Joint Conference on Neural Networks, San Jose, CA, USA, 31 July-5 August 2011.

25. Hitzer, E.; Nitta, T.; Kuroe, Y. Applications of Clifford's geometric algebra. Adv. Appl. Clifford Algebras 2013, 23, 377-404. [CrossRef]

26. Cui, Y.; Takahashi, K.; Hashimoto, M. Remarks on robot controller application of Clifford multi-layer neural networks. In Proceedings of 13th IEEE International Workshop on Advanced Motion Control, Yokohama, Japan, 14-16 March 2014; pp. 410-415. [CrossRef]

27. Takahashi, K. Remarks on self-tuning feedback controller using the Clifford multi-layer neural network. In Proceedings of the 2015 IEEE 20th Conference on Emerging Technologies \& Factory Automation (ETFA), Luxembourg, 8-11 September 2015; pp. 1-6. [CrossRef]

28. Zhu, J.; Sun, J.; Global exponential stability of Clifford-valued recurrent neural networks. Neurocomputing 2016, 173, 685-689. [CrossRef]

29. Liu, Y.; Xu, P.; Lu, J.; Liang, J. Global stability of Clifford-valued recurrent neural networks with time delays. Nonlinear Dyn. 2019, 332, 259-269. [CrossRef]

30. Shen, S.; Li, Y. $S^{p}$-Almost periodic solutions of Clifford-valued fuzzy cellular neural networks with time-varying delays. Neural Process. Lett. 2020, 51, 1749-1769. [CrossRef]

31. Li, Y.; Xiang, J.; Li, B. Globally asymptotic almost automorphic synchronization of Clifford-valued recurrent neural netwirks with delays. IEEE Access 2019, 7, 54946-54957. [CrossRef]

32. Li, B.; Li, Y. Existence and global exponential stability of pseudo almost periodic solution for Clifford-valued neutral high-order Hopfield neural networks with leakage delays. IEEE Access 2019, 7, 150213-150225. [CrossRef]

33. Li, Y.; Xiang, J. Global asymptotic almost periodic synchronization of Clifford-valued CNNs with discrete delays. Complexity 2019, 2019, 6982109. [CrossRef]

34. Li, B.; Li, Y. Existence and global exponential stability of almost automorphic solution for Clifford-valued high-order Hopfield neural networks with leakage delays. Complexity 2019, 2019, 6751806. [CrossRef]

35. Aouiti, C.; Gharbia, I.B. Dynamics behavior for second-order neutral Clifford differential equations: inertial neural networks with mixed delays. Comput. Appl. Math. 2020, 39, 120. [CrossRef]

36. Pecora, L.; Carroll, T. Synchronization in chaotic systems. Phys. Rev. Lett. 1990, 64, 821-824. [CrossRef]

37. Tong, D.; Zhang, L.; Zhou, W.; Zhou, J.; Xu, Y. Asymptotical synchronization for delayed stochastic neural networks with uncertainty via adaptive control. Int. J. Control Autom. Syst. 2016, 14, 706-712. [CrossRef]

38. Guo, Z.; Gong, S.; Yang, S.; Huang, T. Global exponential synchronization of multiple coupled inertial memristive neural networks with time-varying delay via nonlinear coupling. Neural Netw. 2018, 108, 260-271. [CrossRef]

39. Zheng, C.; Cao, J. Robust synchronization of coupled neural networks with mixed delays and uncertain parameters by intermittent pinning control. Neurocomputing 2014, 141, 153-159. [CrossRef]

40. Aouiti, C.; Bessifi, M.; Li, X. Finite-time and fixed-time synchronization of complex-valued recurrent neural networks with discontinuous activations and time-varying delays. Circuits Syst. Signal Process. 2020, 39, 5406-5428. [CrossRef]

41. Yuan, Y.; Song, Q.; Liu, Y.; Alsaadi, F.E. Synchronization of complex-valued neural networks with mixed two additive time-varying delays. Neurocomputing 2019, 332, 149-158. [CrossRef]

42. Mei, J.; Jiang, M.; Wang, X.; Han, J.; Wang, S. Finite-time synchronization of drive-response systems via periodically intermittent adaptive control. J. Frankl. Inst. 2014, 351, 2691-2710. [CrossRef]

43. Yang, X. Can neural networks with arbitrary delays be finite-timely synchronized. Neurocomputing 2014, 143, 275-281. [CrossRef]

44. Liu, Y.; Qin, Y.; Huang, J.; Huang, T.; Yang, X. Finite-time synchronization of complex-valued neural networks with multiple time-varying delays and infinite distributed delays. Neural Process. Lett. 2019, 50, 1773-1787. [CrossRef]

45. Liu, Y.; Huang, J.; Qin, Y.; Yang, X. Finite-time synchronization of complex-valued neural networks with finite-time distributed delays. Neurocomputing 2020, 416, 152-157. [CrossRef]

46. Yang, X.; Cao, J. Finite-time stochastic synchronization of complex networks. Appl. Math. Model. 2010, 34, 3631-3641. [CrossRef]

47. Shen, J.; Cao, J. Finite-time synchronization of coupled neural networks via discontinuous controllers. Cogn. Neurodyn. 2011, 5, 373-385. [CrossRef]

48. Rajchakit, G., Sriraman, R., Lim, C.P., Unyong, B. Existence, uniqueness and global stability of Clifford-valued neutral-type neural networks with time delays. Math. Comput. Simul. 2021. [CrossRef]

49. Rajchakit, G., Sriraman, R., Boonsatit, N., Hammachukiattikul, P., Lim, C.P., Agarwal, P. Global exponential stability of Cliffordvalued neural networks with time-varying delays and impulsive effects. Adv. Differ. Equ. 2021, 208. [CrossRef]

50. Rajchakit, G.; Sriraman, R.; Vignesh, P.; Lim, C.P. Impulsive effects on Clifford-valued neural networks with time-varying delays: An asymptotic stability analysis. Appl. Math. Comput. 2021, 407, 126309. 